\title{
Initial Jet Before the Onset of Effective Electrospinning of Polymeric Nanofibers
}

\author{
Lei $\mathrm{Xu}^{*}{ }^{, 1}$, Wen $\mathrm{Han}^{1}$, Gaofeng Zheng ${ }^{2}$, Dezhi $\mathrm{Wu}^{2,3}$, Xiang Wang ${ }^{2}$ and Daoheng Sun ${ }^{*}, 2$ \\ ${ }^{1}$ School of Mechanical and Electric Engineering, Jingdezhen Ceramic Institute, Jingdezhen 333403, China \\ ${ }^{2}$ School of Physics and Mechanical \& Electrical Engineering, Xiamen University, Xiamen 361005, China \\ ${ }^{3}$ Shenzhen Research Institute of Xiamen University, Shenzhen 518057, China
}

\begin{abstract}
Initial jet usually has a large primary droplet hanging at flying end before the onset of effective electrospinning. The primary droplet is undesired as its diameter is several orders of magnitude higher than that of electrospun nanofibres. A new method is used to derive micro-scaled initial jet and fine primary droplet under applied small-aperture needle by utilizing low solution flow rate and pre-applied electric potential before the extrusion of polymeric solution out of the needle. Small-aperture needle reduces the base of conical pendant, while low solution flow rate prevents a fluidic inrush into conical pendant. The pre-applied electric potential preforms a miniature liquid cone, as an origin of initial jet, within the needle. The conic preformation reduces the formation time of Taylor cone in order to escape from a swollen Taylor cone under a continuous inflow of polymeric solution. The miniature conical pendant grows so acute that it emits fine primary droplet rapidly from its tip with accumulated ions. Carrying primary droplet, thin initial jet experiences axial elongation and circumferential rotation in the space.
\end{abstract}

Keywords: Initial jet, primary droplet, rotation.

\section{INTRODUCTION}

Electrospinning has promising applications in filtering [1-3], sensing [4, 5], and biomedical [6, 7] fields owing to its excellent ability to produce nanofibers with high surface-tovolume ratio. Typically, electrospinning utilizes single needle to spray polymeric solution on conducting substrate for fabrication of nonwoven mats [8-10]. Electrospinning also develops by using coaxial needle $[11,12]$ and needleless surface [13] to emit solutions, by employing semiconducting silicon [14] and even dielectric substrate [15] to collect filaments, and/or by providing various composite solutions [16-18] to functionalize deposits.

Effective electrospinning jet has a combined trajectory of nearly straight line in near field and chaotic curve in far field. The quasilinear segment, next to conical liquid pendant at needle exit, is usually thick owing to a surge of solution from liquid pendant. Sun et al. [19] developed near-field electrospinning to paint a fine pattern by collecting the quasilinear jet; Yang et al. [20] observed a swing of quasilinear segment that enlarges the deposition region of nanofibers on substrate. The curvilinear segment, which functions as a mechanical stretching of electrospinning jet, is globally spiral in the space and locally wavy near the

*Address correspondence to these authors at the (Lei Xu) School of Mechanical and Electric Engineering, Jingdezhen Ceramic Institute, Jingdezhen 333403, China; Tel: 86-798-8465857; Fax: 86-798-8465857; E-mail: charlie1975@163.com and (Daoheng Sun) School of Physics and Mechanical \& Electrical Engineering, Xiamen University, Xiamen 361005, China; Tel: 86-592-2185900; Fax: 86-592-2185900;

E-mail:sundh@xmu.edu.cn substrate. Reneker et al. [21] attributed the spiral to primary whipping instability and the wavy motion to secondary bending instability. Yarin et al. [22] ascribed the instabilities to coulomb interactions on electrospinning jet.

By comparison with much attention on the instabilities, an inevitable primary droplet is seldom mentioned by researchers. Generally, the primary droplet is defined as such a drop hanging at flying end of initial jet before the onset of effective electrospinning. The primary droplet is large and undesired as its diameter is usually several orders of magnitude higher than that of electrospun nanofibres. Nevertheless, it might also play an important role in pulsated electrohydrodynamic printing [23].

The primary droplet originates an initial jet for subsequently effective electrospinning and results from the hysteretic formation of initially critical liquid cone. The hysteresis results in a swollen Taylor cone under a continuous inflow of polymeric solution. Chang et al. utilized a probe tip poking inside liquid pendant to trigger micro-scaled initial jet duly before electrospinning [24]. Here, we characterize the initial jet and primary droplet. A new method is used to generate thin initial jet and fine primary droplet under applied small-aperture needle and low solution flow rate by utilizing pre-applied electric potential prior to the extrusion of PEO solution out of the needle.

\section{EXPERIMENT}

The experiment was well designed for visualized observation by utilizing a typical setup [25], in which a power supply (Dongwen P403-1AC) connected a metal 
needle with the positive and a metal plate with the ground under the needle-to-substrate distance of $10 \mathrm{~cm}$. The needle had a small aperture (i.d. $=0.21 \mathrm{~mm}$, o.d. $=0.41 \mathrm{~mm}$ ) to generate a thin initial jet observed by a high-speed camera (NAC MEMRECAM GX-1). A syringe pump (Harvard 11 Pico Plus) supplied the needle with a $14 \%(\mathrm{w} / \mathrm{w})$ solution of poly(ethylene oxide) (PEO, $\mathrm{MW}=300,000 \mathrm{~g} / \mathrm{mol})$ in deionized water. The electric potential of $10 \mathrm{kV}$ was preapplied prior to the extrusion of PEO solution out of the needle.

\section{RESULTS AND DISCUSSION}

Fig. (1) characterizes three transitional stages for the formation of thin initial jet, which was photographed at the frame rate of $2500 \mathrm{fps}$, under applied polymeric solution flow rate of $10 \mu \mathrm{L} / \mathrm{hr}$. In first stage, the liquid pendant has a miniature cone shape and emits a several-tens-of-micrometer primary droplet. The primary droplet flies so straightly that a thin initial jet connects conical pendant with flying primary droplet owing to the viscoelasticity of polymeric solution as shown in Fig. (1a). Fluidic viscoelasticity as well as nonuniform electric field results in nonlinear flight velocity and acceleration of primary droplet. Although mean flight velocity measures only $1 \mathrm{~mm} / \mathrm{s}$ in the first 562 frames by image processing, instantaneous flight velocity and acceleration approximate $10 \mathrm{~mm} / \mathrm{s}$ and $2 \mathrm{~m} / \mathrm{s}^{2}$ in Frame \#562, respectively.

Fig. (1b) illustrates a combined trajectory of curvilinear elongation and circumferential rotation of initial jet in second stage after bending the initial jet. One observation is that two clear generatrices and a vague directrix, typically in Frame \#567, constitute an envelope cone with a vertex at the tip of conical liquid pendant. The generatrices are plane curves while the directrix is a locus of flying primary droplet. As a reliable interpretation from a viewpoint of orthographic projection, the envelope cone results from a high-speed rotation, not a side-to-side wagging, of curvilinear jet. Another observation is that vertex angle of envelope cone increases from Frames \#563 to \#575 and decreases from Frames \#575 to \#590. The maximum vertex angle of envelope cone approximates the vertex angle of conical liquid pendant in Frame \#575.

Fig. (1c) demonstrates the formation of full-length quasilinear jet when initial jet travels to the substrate in final

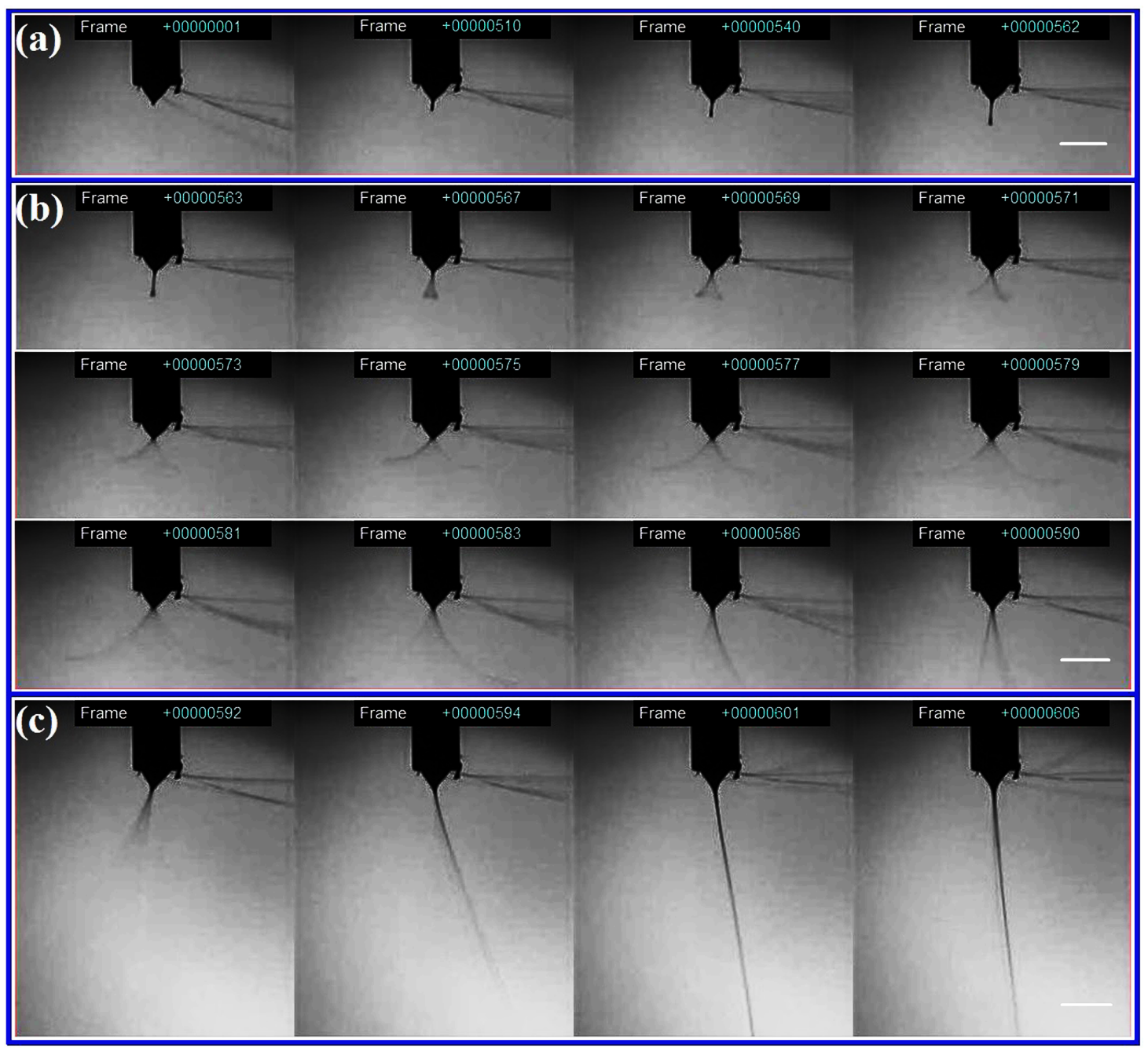

Fig. (1). Thin initial jet experiences three transitional stages before effective electrospinning of nanofibers: (a) straight elongation in near field; (b) the combination of curvilinear elongation and circumferential rotation in the space; and (c) quasilinear connection between conical pendant and substrate. Scale bar: $0.4 \mathrm{~mm}$. 
stage. A physical contact between jet and substrate restrains the flight of primary droplet owing to an adhesive force so that curvilinear jet becomes quasilinear one. Distinguishable path of quasilinear jet indicates its decelerated swing.

Experimental result shows that pre-applied electric potential preforms a conical shape to polymeric solution in the needle. Syringe pump drives polymeric solution out of the needle to generate a liquid pendant adhering to the exit under successive solution supply. Small-aperture needle reduces the base of liquid pendant, while low polymeric solution flow rate prevents an inrush of polymeric solution into liquid pendant. The preformation of liquid cone reduces the formation time of critical Taylor cone in order to escape from a swollen Taylor cone under a continuous inflow of polymeric solution. Hence, the well-designed experiment generates a miniature Taylor cone as an origin of thin initial jet.

Furthermore, carrying injected charges from metal electrode, polymeric solution flows to the tip of conical liquid pendant under pre-applied electric potential. Conical pendant grows so acute that its tip accumulates more and more ions rapidly. Once electric force at the vertex overcomes surface tension force and viscous force after accumulating enough ions, a primary droplet flies out from the tip of conical pendant. A slender liquid column connects flying primary droplet with conical liquid pendant under mechanical stretching owing to the viscoelasticity of polymeric solution. The slender liquid column has the flying primary droplet as a free end with a large mass and the conical liquid pendant as a pinned end. Consequently, a straightly thin initial jet appears as shown in Fig. (1a).

Nevertheless, the interactions of charged ions, as a destabilizing force, impose a physical perturbation on the straight initial jet in non-uniform electric field. The circumferential component of amplifying perturbation may give rise to a rotation of the liquid column at critical slenderness ratio, although the mechanism of circumferential rotation still stays unclear. With the persistent elongation of jet body, the centrifugal effect of large-mass primary droplet bends straight initial jet into curving one owing to an uneven mass distribution. The circumferential rotation of curvilinear jet further generates an envelope cone as shown in Fig. (1b).

Once flying primary droplet reaches a substrate, the substrate restrains the movement of free end due to an adhesive force. The drag effect of substrate attenuates the physical perturbation and retards the circumferential rotation of initial jet. Hence, the initial jet tends to a stable state and gradually becomes a quasilinear jet under mechanical stretching as shown in Fig. (1c).

Subsequently, mechanical tension further stretches the quasilinear jet into fine filament. Coulomb interactions destabilize fine filament again at large slenderness ratio after they overcome the stabilizing viscous effect. The circumferential and axial components of amplifying perturbation make the fine filament into a spiral filament, which is the well-known whipping. The spiral filament is not photographed in this experiment, as it is too fine to distinguish by applied camera.
As a result, the well-designed experiment gives rise to thin initial jet by utilizing pre-applied electric potential, small-aperture needle, and low polymeric solution flow rate. The thin initial jet experiences axial elongation and circumferential rotation in the space until it travels to the substrate. Nevertheless, electrospinning process may also result in thickly initial jet and large primary droplet by utilizing large-aperture needle, high polymeric solution flow rate, or post-applied electric potential after the extrusion of polymeric solution out of the needle. Resulting from a swollen liquid pendant under a surge of polymeric solution from the needle, the thickly initial jet only experiences axial elongation in the space.

Fig. (2) characterizes a thickly initial jet and large primary droplet under applied large-aperture needle (i.d. = $0.60 \mathrm{~mm}$, o.d. $=0.90 \mathrm{~mm}$ ), high polymeric solution flow rate of $80 \mu \mathrm{L} / \mathrm{hr}$, and pre-applied electric potential of $10 \mathrm{kV}$. It is observed that the thickly initial jet only experiences a straight projection. The thickly initial jet is so robust owing to stabilizing viscous and inertial effects at small slenderness ratio that the ionic interactions cannot perturb straight flight of initial jet.

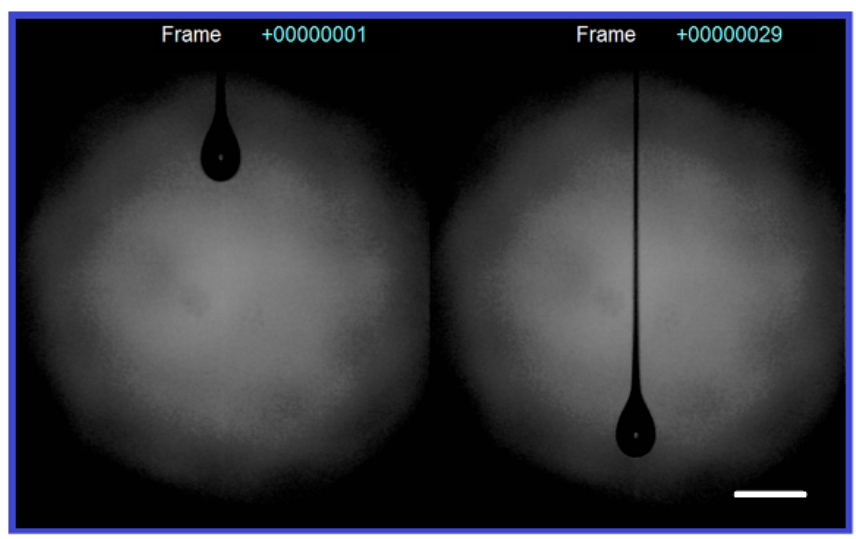

Fig. (2). Thickly initial jet only experiences a straight flight under applied large-aperture needle and high polymeric solution flow rate. Scale bar: $1 \mathrm{~mm}$.

\section{CONCLUSION}

A miniature conical pendant is preformed under preapplied electric potential, small-aperture needle, and low polymeric solution flow rate. The preformation of conical pendant reduces the formation time of critical Taylor cone so that the miniature conical pendant emits fine primary droplet and thin initial jet. Carrying primary droplet at flying end, thin initial jet flies to the substrate. Coulomb interactions perturb the straight flight of thin initial jet to generate the circumferential rotation in the space. Nevertheless, thickly initial jet results in large primary droplet with large mass so that it only experiences axial elongation before effective electrospinning.

\section{CONFLICT OF INTEREST}

The authors confirm that this article content has no conflict of interest. 


\section{ACKNOWLEDGEMENTS}

Project supported by the National Natural Science Foundation of China (51305373), the Foundation of Education Bureau of Jiangxi Province (GJJ13634), and the Science and Technology Program of Shenzhen City (JCYJ20120615161609592), China.

\section{REFERENCES}

[1] A. Mataram, A.F. Ismail. "Structural and characterization polyacrylonitrile nanofiber for air filtration assembled by electrospinning". Prosiding Seminar Nasional Sains dan Teknologi, vol. 1, No. 1, 2011.

[2] H. Park. "Electrospinning of nanofibers for filtration media". Ph.D. dissertation. University of Florida, USA, 2010.

[3] G. Lang, S. Jokisch, T. Scheibel. "Air filter devices including nonwoven meshes of electrospun recombinant spider silk proteins". Journal of Visualized Experiments, vol. 75, e50492, 2013.

[4] E. Zampetti, A. Macagnano, A. Bearzotti. "Gas sensor based on photoconductive electrospun titania nanofibres operating at room temperature". Journal of Nanoparticle Research, vol. 15, pp. 1-8, 2013.

[5] V. Modafferi, G. Panzera, A. Donato, P.L. Antonucci, C. Cannilla, N. Donato, D. Spadaro, G. Neri. "Highly sensitive ammonia resistive sensor based on electrospun V2O5 fibers". Sensors and Actuators B: Chemical, vol. 163, pp. 61-68, 2012.

[6] A.J. Meinel, O. Germershaus, T. Luhmann, H.P. Merkle, L. Meinel. "Electrospun matrices for localized drug delivery: Current technologies and selected biomedical applications". European Journal of Pharmaceutics and Biopharmaceutics, vol. 81, pp. 1-13, 2012.

[7] G. Panthi, N.A.M. Barakat, P. Risal, A. Yousef, B. Pant, A.R. Unnithan, and H.Y. Kim, "Preparation and characterization of Nylon-6/gelatin composite nanofibers via electrospinning for biomedical applications", Fibers and Polymers, vol. 14, pp. 718723, 2013.

[8] W. Zhou, H. Huang, S. Du, Y. Huo, J. He, and S. Cui, "Layer-bylayer structured membranes of silk fibroin and polyethylenimine on electrospun silm fibroin nanofibers", The Open Materials Science Journal, vol. 8, pp. 81-86, 2014.

[9] J. Liu, W. Shou, L. Zhang, W. Liang, D.W. Huang, and Y. Liu, "A scaling model for predicting the gas-channel formation period in crater-like electrospinning of nanofibers", The Open Materials Science Journal, vol. 8, pp. 114-118, 2014.

[10] C. Fang, Y. Jiang, and L. Xu, "Impact of overlapping collector on orientated deposition of electrospun nanofibers", The Open Materials Science Journal, vol. 8, pp. 119-121, 2014.

[11] W. Huang, T. Zou, S. Li, J. Jing, X. Xia, and X. Lin, "Drug-loaded zein nanofibers prepared using a modified coaxial electrospinning process", AAPS PharmSciTech, vol. 14, pp. 675-681, 2013.
[12] J. Cao, T. Zhang, F. Li, H. Yang, and S. Liu, "Enhanced ethanol sensing of $\mathrm{SnO}_{2}$ hollow micro/nanofibers fabricated by coaxial electrospinning", New Journal of Chemistry, vol. 37, pp. 20312036, 2013.

[13] X. Wang, X. Hu, X. Qiu, X. Huang, D. Wu, and D. Sun, "An improved tip-less electrospinning with strip-distributed solution delivery for massive production of uniform polymer nanofibers", Materials Letters, vol. 99, pp. 21-23, 2013.

[14] G. Zheng, W. Li, X. Wang, D. Wu, D. Sun, and L. Lin, "Precision deposition of a nanofibre by near-field electrospinning", Journal of Physics D: Applied Physics, vol.43, 415501, 2010.

[15] J. Lee, S.Y. Lee, J. Jang, Y.H. Jeong, and D.W. Cho, "Fabrication of patterned nanofibrous mats using direct-write electrospinning", Langmuir, vol. 28, pp. 7267-7275, 2012.

[16] S. Agarwal, A. Greiner, and J.H. Wendorff, "Functional materials by electrospinning of polymers", Progress in Polymer Science, vol. 38, pp. 963-991, 2013.

[17] Z.H. Liu, C.T. Pan, L.W. Lin, and H.W. Lai, "Piezoelectric properties of PVDF/MWCNT nanofiber using near-field electrospinning", Sensors and Actuators A: Physical, vol. 193, pp. $13-24,2013$

[18] T.M. Araujo, S. Sinha-Ray, A. Peqoretti, and A.L. Yarin, "Electrospinning of a blend of a liquid crystalline polymer with poly(ethylene oxide): Vectran nanofiber mats and their mechanical properties", Journal of Materials Chemistry C, vol. 1, pp. 351-358, 2013.

[19] D. Sun, C. Chang, S. Li, and L. Lin, "Near-Field Electrospinning". Nano Letters, vol. 6, pp. 839-842, 2006.

[20] Y. Yang, Z. Jia, L. Hou, Q. Li, L. Wang, and Z. Guan, "Controlled deposition of electrospinning jet by electric field distribution from an insulating material surrounding the barrel of the polymer solution", IEEE Transactions on Dielectrics and Electrical Insulation, vol. 15, pp. 269-276, 2008.

[21] D.H. Reneker, A.L. Yarin, H. Fong, and S. Koombhongse, "Bending instability of electrically charged liquid jets of polymer solutions in electrospinning", Journal of Applied Physics, vol. 87, pp. 4531-4547, 2000.

[22] A.L. Yarin, S. Koombhongse, and D.H. Reneker, "Bending instability in electrospinning of nanofibers", Journal of Applied Physics, vol. 89, pp. 3018-3026, 2001.

[23] L. Xu, and D. Sun, "Electrohydrodynamic printing under applied pole-type nozzle configuration", Applied Physics Letters, vol. 102, 024101, 2013.

[24] C. Chang, K. Limkrailassiri, and L. Lin. "Continuous near-field electrospinning for large area deposition of orderly nanofiber patterns". Applied Physics Letters, vol. 93, 123111, 2008.

[25] V. Pillay, C. Dott, Y.E. Choonara, C. Tyagi, L. Tomar, P. Kumar, L.C. du Toit, and V.M.K. Ndesendo, "A review of the effect of processing variables on the fabrication of electrospun nanofibers for drug delivery applications", Journal of Nanomaterials, 789289, 2013. 